\title{
Estudo de caso: neuropatia auditiva
}

\section{Auditory neuropathy: a study case}

\author{
Viviane M. Parra' ${ }^{1}$ Carla G. Matas ${ }^{2}$, Ivone F. Neves ${ }^{3}$
}

Palavras-chave: neuropatia auditiva, audição, testes auditivos.

Key words: auditory neuropathy, hearing, hearing tests.

\section{Resumo / Summary}

\begin{abstract}
A ualmente, podemos complementar os resultados encontrados na audiometria convencional com os obtidos nos exames eletrofisiológicos, o que nos permite diagnosticar não apenas uma perda auditiva periférica, mas também diferenciar uma perda auditiva coclear da neural, ou central. Isto nos conduziu a um grupo novo de pacientes que apresentava alteração na sincronia neural com função normal das células ciliadas externas. Esta patologia é conhecida como Neuropatia Auditiva. 0 objetivo deste estudo é descrever as características audiológicas de um paciente com Neuropatia Auditiva atendido no Centro de Docência e Pesquisa em Fonoaudiologia da Faculdade de Medicina da Universidade de São Paulo (FMUSP), correlacionando os achados com os encontrados na literatura. 0 caso descrito é de um indivíduo de 24 anos de idade, com o diagnóstico de Neuropatia Auditiva, atendido no ano de 2001 no Centro de Docência e Pesquisa em Fonoaudiologia da FMUSP. Foram realizados anamnese, audiometria tonal e vocal, medidas de imitância acústica, Emissões O toacústicas (EOAs) e Audiometria de Tronco Encefálico (ABR). Observamos no caso estudado a incompatibilidade de resultados entre audiometria tonal e testes de inteligibilidade de fala, com os exames objetivos, EOAs e $A B R$, onde observou-se perda auditiva com importante alteração nos testes de inteligibilidade de fala, EO As presentes e $A B R$ anormal. Estes dados sugerem função coclear normal e alteração da sincronia neural.
\end{abstract}

\begin{abstract}
N owadays, in our clinic, we can complement the conventional audiometry results with electrophysiologic exams. Not only does allow us to diagnose a peripheral hearing loss but also to differentiate cochlear, neural or central hearing loss. It led us to a new group of patients that presented changes in neural synchrony, with normal functions of the outer ear hair cells. This pathology is known as Auditory Neuropathy. Our study purpose is describe the audiologist characteristic of a patient with auditory neuropathy attended at "Centro de Docência e Pesquisa em Fonoaudiologia da Faculdade de Medicina da Universidade de São Paulo", correlating the study data with literature. The patient described is a 24 year-old person, with auditory neuropathy diagnosis, evaluated in our center in 2001. Interview, tonal audiometry thresholds, speech recognition, timpanometry, otoacustic emissions (OAEs) and auditory brainstem responses (ABR) were applied. In this case we observed incompatible results in the tonal audiometric threshold and speech recognition tests, with objectives tests like OAEs and $A B R$, in which we found a hearing loss with important changes in the speech recognition test, of $O A E s$ present and abnormal ABR. These data suggest a normal cochlear function and abnormal neural synchrony.
\end{abstract}

\footnotetext{
${ }^{1}$ Especializanda em Audiologia Clínica da FMUSP.

${ }^{2}$ Professora Doutora do Curso de Fonoaudiologia da FMUSP.

${ }^{3}$ Mestranda do Curso de Fonoaudiologia da FMUSP.

Instituição: Curso de Fonoaudiologia da Faculdade de Medicina de São Paulo.

Endereço para correspondência: Viviane M. Parra - R. Aimberê, 311 Perdizes São Paulo SP 05018-010

Tel (0xx11) 3872-9662 - E-mail: viviane.parra@ig.com.br ou carlagmatas@globo.com.br ou ifneves@usp.br

Artigo recebido em 26 de dezembro de 2001. Artigo aceito em 26 de agosto de 2002.
} 


\section{INTRODUÇÃO}

Um indivíduo adulto compareceu à Clínica de Audiologia do Centro de Docência e Pesquisa em Fonoaudiologia da FMUSP para realização de avaliação audiológica, porém o fato de haver uma incompatibilidade de resultados entre audiometria tonal, audiometria vocal, emissões otoacústicas evocadas (EOAs) e Audiometria de Tronco Encefálico ( $A B R$ ) nos alertou para o fato daquele indivíduo apresentar uma Neuropatia Auditiva.

Segundo Matas et al. (1998), a ABR é um exame objetivo da audição que avalia a integridade da via auditiva. Este procedimento não tem o objetivo de substituir outros procedimentos audiológicos de rotina, mas sim, complementá-los no diagnóstico da deficiência auditiva.

As ondas encontradas no registro da $A B R$ são geradas por uma ou mais estruturas ao longo da via auditiva. Uma das classificações mais utilizadas é a de Möller, Jannetta, Bennett \& Möller (1981), na qual estão descritos os sítios geradores: onda I - porção distal ao tronco encefálico do nervo auditivo, onda II - porção proximal, onda III - núcleo coclear, onda IV - complexo olivar superior, onda V lemnisco lateral, onda VI colículo inferior, onda VII - corpo geniculado medial.

As EOAs são sons sub-audíveis gerados na cóclea normal e coletadas no meato acústico externo. Esses sons são gerados a partir da vibração da cóclea normal (células ciliadas externas) espontaneamente ou por estimulação sonora, são retransmitidos de forma eferente através do sistema tímpano-ossicular e captadas no meato acústico externo.

Segundo Lopes Filho (1997) foram estudados dois tipos básicos de EOAs, as EOAs espontâneas e as EOAs evocadas. As EO As espontâneas são energias acústicas de banda estreita, provenientes da cóclea, sem que haja estimulação específica. As Emissões 0 toacústicas Evocadas Transientes (EOAT) são respostas obtidas a partir de breve estimulação da cóclea, utilizando-se de "clicks" que são estímulos acústicos transitórios de curta duração e com faixa de freqüência abrangente. As Emissões O toacústicas por Produto de Distorção (EOAPD) são respostas obtidas no meato acústico externo, originando-se da cóclea pela interação não linear de dois tons puros aplicados simultaneamente.

É importante lembrar que as EOAs nos permitem verificar o estado funcional da cóclea mas não têm relação direta com o limiar fisiológico. O wens et al. (1993) afirmou que qualquer alteração da transmissão dessa energia sonora poderá acarretar diminuição ou ausência de respostas comprometendo assim a análise do exame. Portanto, as medidas de imitância acústica são indispensáveis na hora do diagnóstico, descartando um comprometimento condutivo que possa alterar o resultado do exame.

Antigamente 0 diagnóstico da deficiência auditiva baseava-se nos resultados obtidos na ABR. Com a introdução das EOAs, complementando a bateria de exames audiológicos, houve a possibilidade de se identificar um grupo novo de pacientes que apresentavam alteração na sincronia neural com função normal das células ciliadas externas. Esta patologia é conhecida como Neuropatia Auditiva.

Hood (1998a) definiu a Neuropatia Auditiva como o termo utilizado para descrever condições que são encontradas em pacientes de todas as idades, adultos ou crianças, com audição compatível com função coclear normal e alteração na sincronia neural. Estas características são observadas nos testes audiológicos com EOAs normais e ABR ausentes ou severamente comprometida.

0 objetivo deste estudo é descrever as características audiológicas de um paciente com Neuropatia Auditiva atendido no Centro de Docência e Pesquisa em Fonoaudiologia da FMUSP, correlacionando os achados com os encontrados na literatura.

\section{MATERIAL E MÉTODO}

0 caso descrito é de um adulto, 24 anos de idade, com o diagnóstico de Neuropatia Auditiva, atendido no ano de 2001 no Centro de Docência e Pesquisa em Fonoaudiologia da FMUSP.

Inicialmente realizou-se anamnese para obter informações sobre as dificuldades auditivas, antecedentes para a deficiência auditiva e impacto da perda auditiva na sua qualidade de vida e nos aspectos psicossociais.

A avaliação audiológica foi realizada em cabine acústica e os procedimentos empregados foram:

- Audiometria Convencional, nos moldes adotados por Mangabeira Albernaz et al. (1981), realizada com o audiômetro Modelo GSI 16, da marca Grason-Stadler.

- Medidas de Imitância Acústica, realizada com o Imitânciometro Modelo GSI-33, da marca Grason-Stadler.

- Emissões O toacústicas Evocadas Transientes, realizada com o Analisador de Emissões Cocleares O todynamics, ILO 88 - Versão 4.2, o qual dispõe dos parâmetros de registro segundo proposto por Kemp (1990) e as Emissões O toacústicas Evocadas por Produto de Distorção, com 0 equipamento ILO 92.

A pós a avaliação audiológica, realizou-se a avaliação eletrofisiológica da audição empregando-se como procedimento a $A B R$, realizada com o equipamento Modelo Spirit da marca Nicolet.

\section{RESULTADOS}

\section{Anamnese}

A queixa trazida pelo paciente foi de dificuldade de 
compreensão de fala. 0 paciente referiu ouvir as palavras, porém não era capaz de compreendê-las em diversas situações, como por exemplo, conversa entre dois indivíduos, rádio, televisão, telefone e principalmente na presença de ruído competitivo.

Segundo ele, a dificuldade de compreensão de fala deu-se progressivamente, a partir dos 14 anos de idade, quando sofreu um traumatismo crânio-encefálico.

\section{Audiometria Tonal e Vocal}

$\mathrm{Na}$ audiometria tonal observou-se perda auditiva neurossensorial de grau leve a moderadamente severo na orelha esquerda e de grau leve a severo na orelha direita.

0 paciente encontrou muita dificuldade na realização da audiometria vocal, apresentando respostas incompatíveis com a audiometria tonal no teste de Limiar de Reconhecimento de Fala (SRT) e baixo Índice de Reconhecimento de Fala (IPRF) em ambas as orelhas.

\begin{tabular}{lcc}
\hline & Audiometria Vocal & \\
& OD & OE \\
\hline SRTc/Masc p/OE & $85 \mathrm{~dB}$ & $55 \mathrm{~dB}$ \\
IPRF(dissílabos) & $4 \%-90 \mathrm{~dB}$ & $8 \%-90 \mathrm{~dB}$ \\
\hline
\end{tabular}

\section{Medidas de I mitância Acústica} bilaterais.

Timpanometria: Curvas timpanométricas normais

Pesquisa dos Reflexos Acústicos: reflexos estapedianos contra e ipsilaterais ausentes bilateralmente.

Emissões O toacústicas Evocadas Transientes e por Produto de Distorção

Observou-se presença de Emissões Otoacústicas Transientes e por Produto de Distorção bilateralmente.

\section{Audiometria de Tronco Encefálico (ABR)}

Na ABR observou-se ausência de Ondas I, III e V a $100 \mathrm{~dB}$ para "clicks" na orelha direita e presença das O ndas I e III a 100dB para "clicks" na orelha esquerda, com latências absolutas e interpicos aumentados.

\section{Tomografia Computadorizada dos Ossos Temporais}

O paciente passou por avaliação otorrinolaringológica onde foi solicitada a tomografia computadorizada dos ossos temporais. Foram encontrado os seguintes resultados:

- Mastóides normopneumatizados.

- Conduto auditivo externo, esporão, antro, cadeia ossicular, cóclea, vestíbulo, canais semi-circulares e conduto auditivo interno sem alterações bilateralmente.

- Conclusão: Exame normal.

\section{Exame Neurológico}

- Estado de Consciência: alerta, consciente, orientado têmporo-espacialmente, auto e alopsiquicamente.

- Força muscular: grau V, global e simétrico.

- Reflexos profundos: grau II, simétrico.

- Reflexos superficiais: presentes e normais.

- Provas cerebelares: normais.

- Marcha e equilíbrio sem alteração.

- Nervos cranianos: hipoacusia bilateral, mais intensa à direita. Restantes dos nervos cranianos sem alteração.

\section{DISCUSSÃO}

Diante dos resultados descritos acima constatamos uma perda auditiva neurossensorial de grau moderado na

\section{Audiograma}

Orelha Direlta

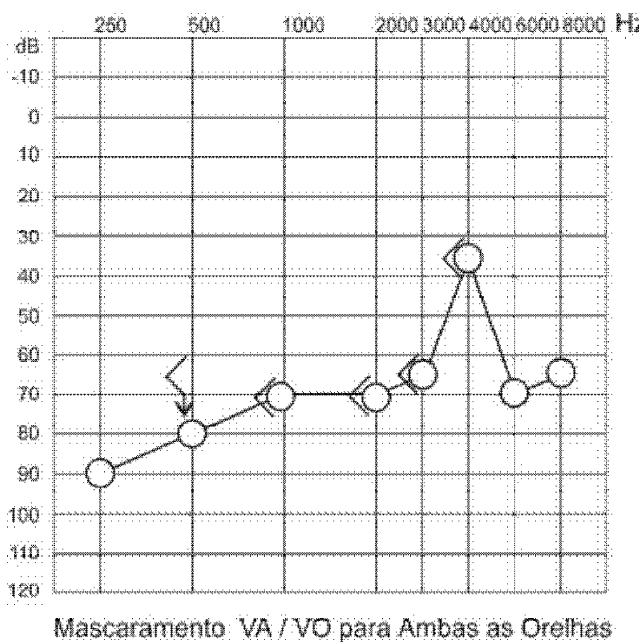

Orelha Esquerda

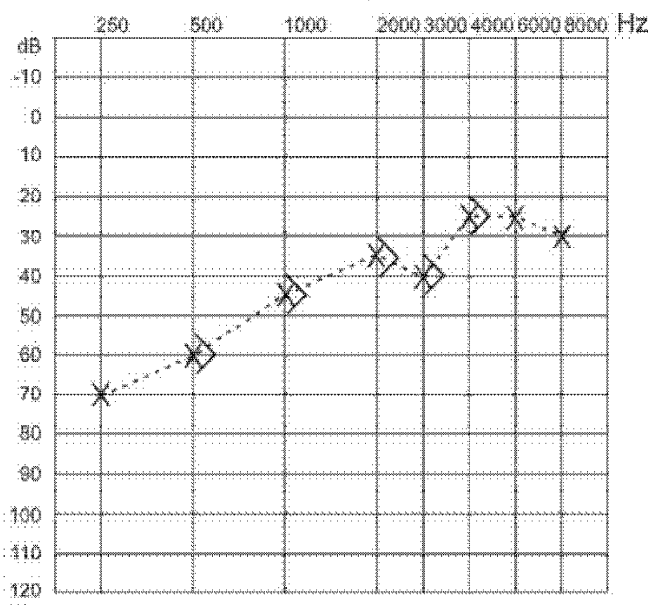




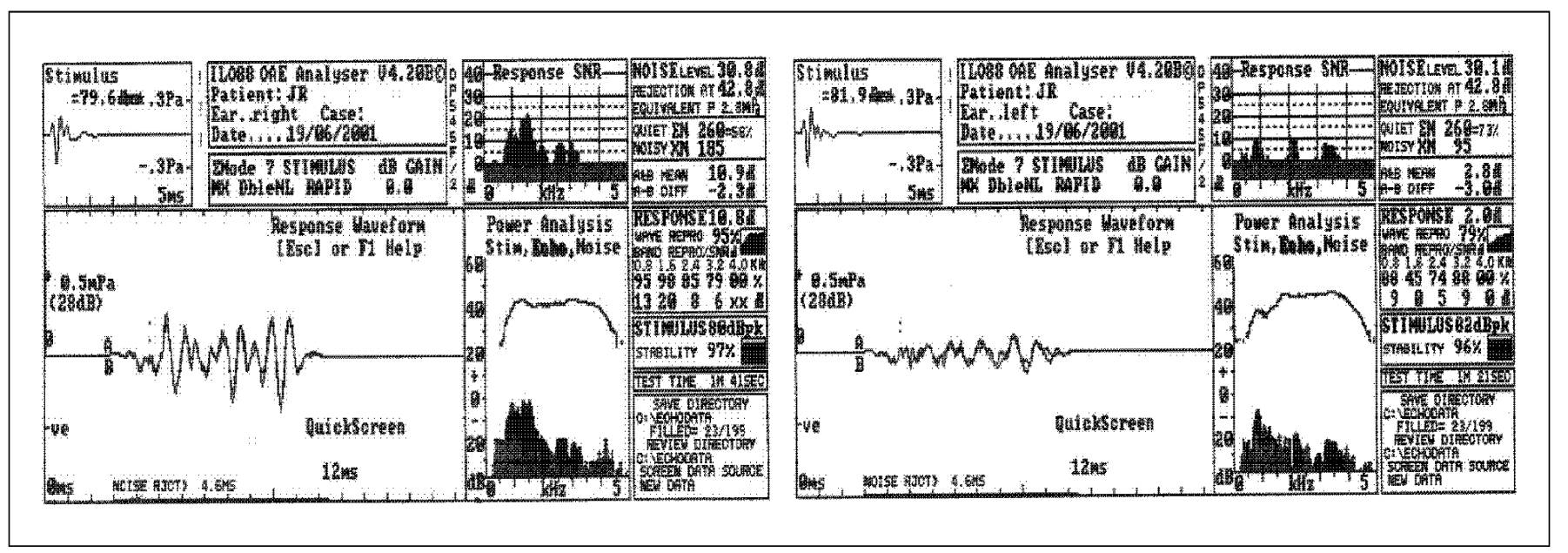

Figura 1. Emissões Otoacústicas Transiente do paciente J.R.

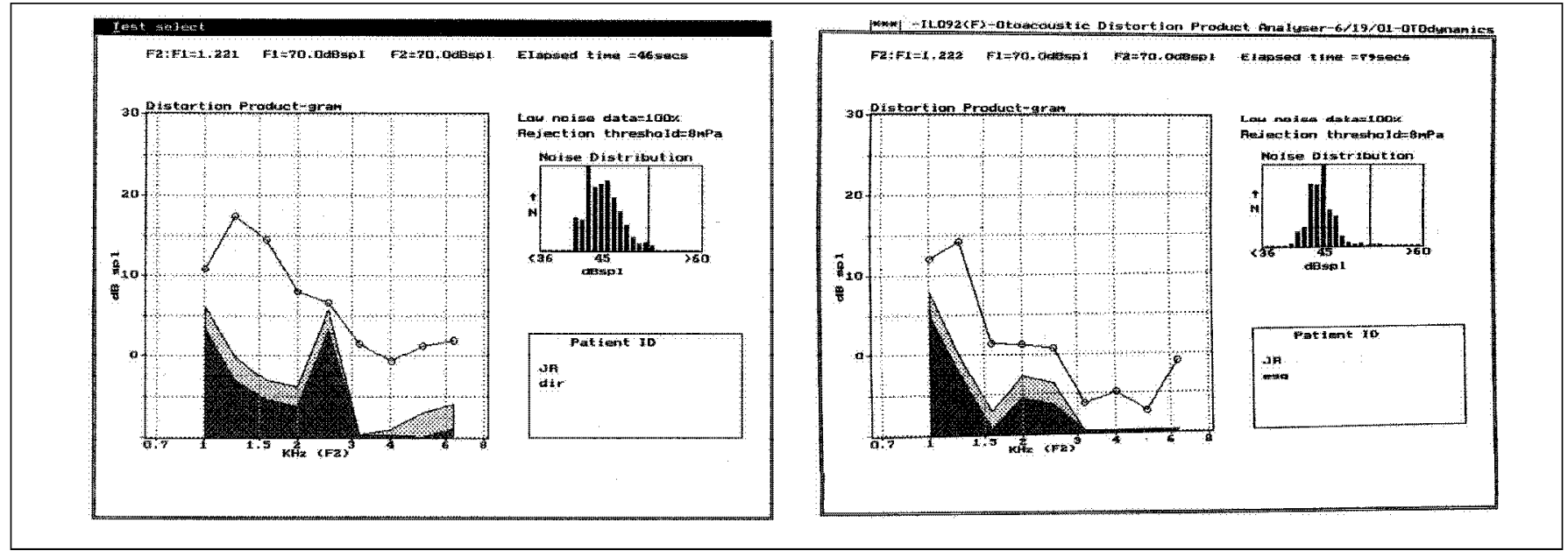

Figura 2. Emissões Otoacústicas por Produto de Dirtorção do paciente J.R.

orelha direita e de grau severo na esquerda, com incompatibilidade de resultados entre a audiometria tonal e a audiometria vocal, já que apresentou um desempenho ruim nos testes de inteligibilidade de fala. Outro dado importante foi a incompatibilidade entre os resultados obtidos na avaliação audiológica convencional (audiometria e medidas de imitância acústica) e os obtidos nos exames objetivos de audição (EO As presentes e ABR alterado).

Hood (1999) afirma a importância dos testes audiológicos objetivos para o diagnóstico diferencial das perdas auditivas e afirma que estes, somados a audiometria tonal e vocal serão mais eficazes, principalmente para o diagnóstico da neuropatia auditiva. Para isso, cita os seguintes testes:

\section{Medidas de Imitância Acústica}

Sua resposta depende da orelha média, da função aferente do oitavo nervo craniano e da ativação eferente do nervo facial. Pacientes com Neuropatia Auditiva geralmente apresentam curva timpanométrica normal e ausência de reflexos acústicos do músculo estapédio.

\section{Emissões Otoacústicas Transientes e Efeito de Supressão}

A presença de respostas das EOAT são associadas com função coclear normal. 0 efeito de supressão ocorre como resultado da ativação da via eferente medial descendente do feixe olivococlear. Pacientes com Neuropatia Auditiva apresentam EOAT presentes e ausência de supressão. 


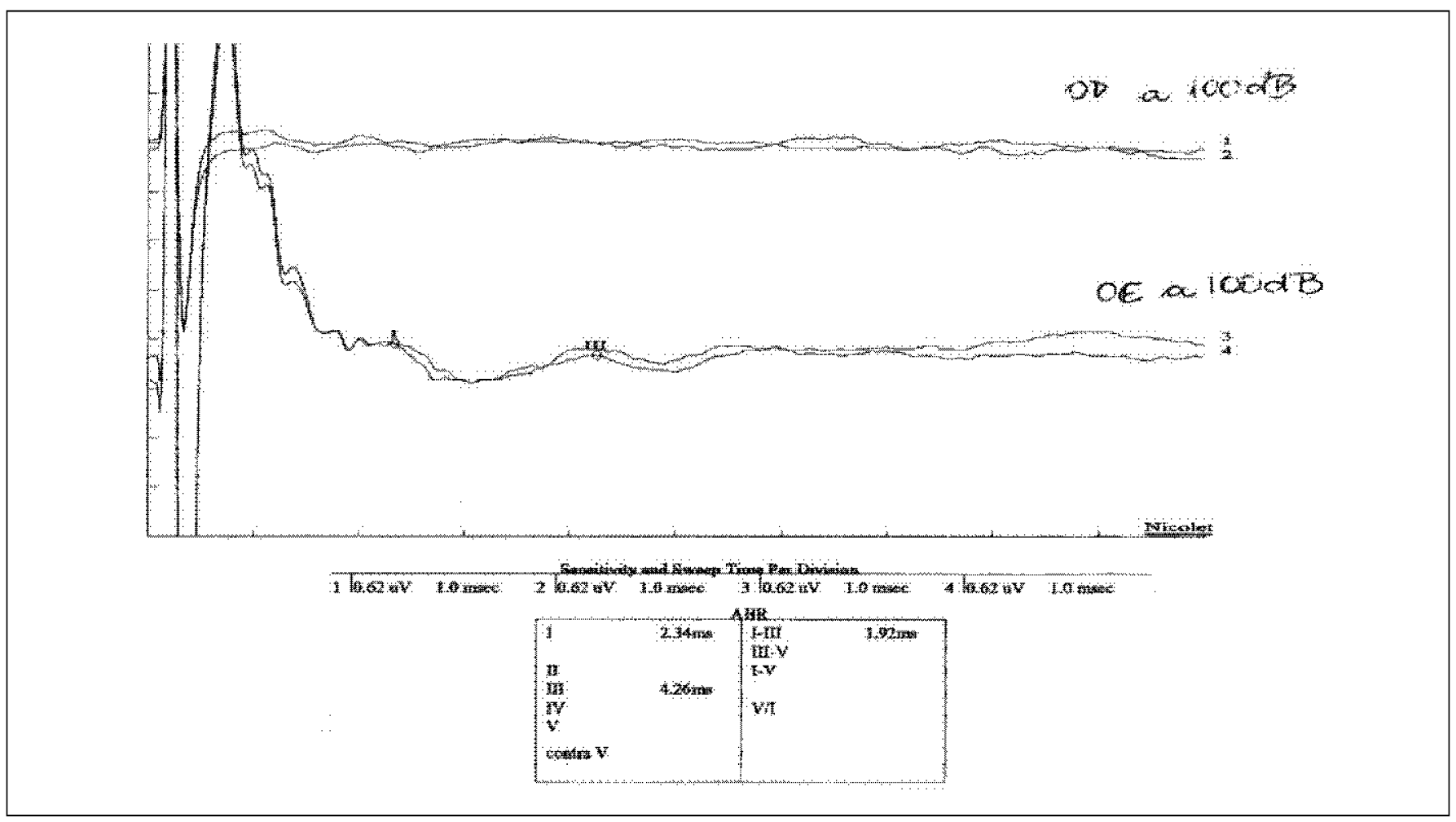

Figura 3. Audiometria de Tronco Encefálico do paciente J.R.

\section{Audiometria de Tronco Encefálico (ABR)}

Avalia a via auditiva a partir do VIII nervo craniano até o lemnisco lateral - Tronco Encefálico. Pacientes com Neuropatia Auditiva apresentam ABR ausente ou severamente comprometida.

Hood (1999) descreveu casos de neuropatia auditiva, com o objetivo de mostrar a importância dos testes eletrofisiológicos para o seu diagnóstico. Um dos casos diagnosticados como Neuropatia Auditiva era de um adolescente com audiometria tonal indicando perda auditiva neurossensorial de grau moderado em freqüências agudas, reconhecimento de fala pobre e ausente com ruído competitivo, timpanometria normal, reflexos acústicos ipsi e contralaterais ausentes, EOAT presentes, efeito de supressão ausente, $A B R$ com ausência de respostas, presença de microfonismo coclear (inversão do traçado com a mudança de polaridade do estímulo), potencial de média latência ausente e respostas corticais normais.

Em outro estudo, Hood (1998b) relatou um caso de uma criança de 3 anos onde observou-se EOAs presentes bilateralmente e alteração nas respostas do ABR, confirmando o diagnóstico de neuropatia auditiva.

Starr et al. (1996) estudaram as habilidades auditivas de pacientes com neuropatia auditiva e relataram que em 10 pacientes ( 5 adultos e 5 crianças) todos apresentaram perda auditiva para tom puro variando de grau leve a severo, com alteração no testes de inteligibilidade de fala, $A B R$ ausente ou gravemente anormal e EO As normais.
Os resultados encontrados por Starr et al. (1996) e Hood (1988a, 1998b, 1999) foram semelhantes aos obtidos no presente estudo, já que todos tinham como características básicas a incompatibilidade de resultados, caracterizado por baixo índice de reconhecimento de fala, incompatível com a audiometria tonal, presença de EOAs e ABR severamente comprometida.

\section{CONCLUSÃO}

Observou-se no caso estudado a incompatibilidade de resultados entre audiometria tonal e testes de inteligibilidade de fala, com os resultados dos exames objetivos (EOAs e ABR), apresentando perda auditiva com importante alteração nos testes de inteligibilidade de fala, EOAS presentes e ABR anormal. Estes dados sugerem função coclear normal e alteração da sincronia neural.

Ressalta-se a importância da realização de uma bateria completa de exames audiológicos, o que possibilita 0 diagnóstico não apenas uma perda auditiva periférica, mas também diferenciação entre uma perda auditiva coclear, neural e central.

Procurou-se através deste estudo descrever os resultados encontrados nas avaliações audiológicas e eletrofisiológicas de um indivíduo com Neuropatia Auditiva, correlacionando com os trabalhos já realizados, a fim de realizar-se um diagnóstico mais eficiente dessa afecção, ainda pouco conhecida. 


\section{REFERÊNCIASBIBLIOGRÁFICAS}

1. Hood LJ. Auditory Neuropathy: What is it and what can we do about it? The Hearing Journal 1998a;51(8):10-7.

2. Hood LJ. Update on Clinical Application of the Auditory Brainstem Response and Otoacoustic Emissions. New Orleans, L.A., agosto 1998b. Disponível em: http:/ www. LHood@LSUMC.EDU.

3. Hood LJ. A Review of Objective Methods of Evaluating Auditory Neural Pathways. The Laryngoscope 1999;109:1745-8.

4. Lopes Filho $0 \&$ Carlos RC. Emissões Otoacústicas. In: Campiotto $A R$, LevyC, Holzhein D, Vicente LCC, Castiglioni M, Redondo MC, Anelli W. Tratado do Fonoaudiologia. Brasil: ed. Roca; 1997. p.222-37.

5. Mangabeira Albernaz $\mathrm{P}$, Mangabeira Albernaz PL, Mangabeira Albernaz LG, Mangabeira Albernaz Fo P. Otorrinolaringologia Prática. 10ª ed. São Paulo: Sarvier; 1981.
6. Matas CG, Frazz MM, Munhoz MSL. Aplicação do Potencial Auditivo de Tronco Encefálico em Audiologia Pediátrica. In: Basseto MCA, Brock R, Wajntein R. Neonatologia. Um Convite à Atuação Fonoaudiológica. São Paulo:Lovise; 1998. p. 301-10.

7. Möller AR, Jannetta $P$, Bennett M, Möller MB. Intracranially Recorded Responses from Human Auditory Nerve: New Insights Into the Oringin of Brainstem Evoked Potencials. Eletroencephalography and Clinical Neurophysiology 1981;52:18-27.

8. Owens JJ, Mccoy MJ, Lonsbury-Mrtin BL, Martin GK. Otoacustic Emissions in Children with Normal Ears, Middle Ear Dysfunction, and Ventilating Tubes. Am J Otol 1993;14(1):34-40.

9. Starr A, Picton TW, Sininger $\mathrm{Y}, \mathrm{Hood} \mathrm{LJ}$, Berlin $\mathrm{Cl}$. Auditory Neuropathy. Brain 1996;119:741-58. 